\title{
Aspectos sensoriais e a seletividade alimentar da criança com transtorno do espectro autista: um estudo de revisão integrativa
}

\author{
Sensory aspects and dietary selectivity of children with autism spectrum disorder: an integrative \\ review study
}

Aspectos sensoriales y selectividad alimentaria del niño con trastorno del espectro autista: un estudio de revisión integrativa

Recebido: 31/07/2021 | Revisado: 10/08/2021 | Aceito: 16/08/2021 | Publicado: 18/08/2021

\author{
Ávyla Germano Santos Silva \\ ORCID: https://orcid.org/0000-0003-2950-3296 \\ Centro Universitário de João Pessoa, Brasil \\ E-mail: avyllag@gmail.com \\ Simone Pereira Lins Chaves \\ ORCID: https://orcid.org/0000.0002.0288.0721 \\ Centro Universitário de João Pessoa, Brasil \\ E-mail: splfono@msn.com \\ Larissa Nadjara Alves Almeida \\ ORCID: https://orcid.org/0000-0002-6818-3398 \\ Cento Universitário de João Pessoa, Brasil \\ E-mail: larissa_nadjara@hotmail.com \\ Ruth Lopes do Nascismento \\ ORCID: https://orcid.org/0000-0002-8974-8263 \\ Centro Universitário de João Pessoa, Brasil \\ E-mail: ruth.nascimento@unipe.edu.br \\ Marcela Leiros Maciel Macêdo \\ ORCID: https://orcid.org/0000-0002-4991-2225 \\ Universidade Federal da Paraíba, Brasil \\ E-mail: marcelaleiros@hotmail.com \\ Adriana Queiroga Sarmento \\ ORCID: https://orcid.org/0000.0002.8714.7923 \\ Universidade Federal da Paraíba, Brasil \\ E-mail: aqsguerra@gmail.com
}

\begin{abstract}
Resumo
Introdução: O Transtorno do Espectro Autista (TEA) é considerado um transtorno do neurodesenvolvimento que afeta a comunicação social e comportamentos, que são restritos, persistentes ou estereotipados, incluindo as alterações no processamento sensorial, de modo hipo ou hiper-reativa. Essas disfunções sensoriais podem provocar problemas na alimentação, sendo a seletividade alimentar a mais comum em crianças com TEA, que é determinada pela rejeição alimentar, resistência ao novo e limitação no repertório alimentar. Objetivo: Identificar os aspectos sensoriais e sua interferência na seletividade alimentar das crianças com Transtorno do Espectro Autista. Métodos: Consiste em uma revisão integrativa, utilizando as seguintes bases de dados eletrônica: Lilacs (Literaturac Latino-Americana e do Caribe em Ciências da Saúde), PubMed (U.S. National Library of Medicine) e SciELO (Scientific Electronic Library Online) através de cruzamento dos seguintes descritores, nos idiomas português e inglês: Seletividade alimentar/ Food Fussiness; Comportamento alimentar/Feeding Behavior; Transtorno do Espetro Autista/Autism Spectrum Disorder; Autismo/Autism; Autismo infantil/Autistic Disorder, utilizando o operador booleano AND. Resultados: Obteve-se um universo de 818 artigos, após os critérios elegíveis foram selecionados 39 artigos. Por fim, após leitura dos artigos na íntegra, resultou em 9 artigos compondo o estudo. Conclusão: A literatura científica demonstra que as crianças com Transtorno do Espectro Autista apresentam alterações sensoriais, como: sensibilidade sensorial oral, tátil e olfativa. Tendo como consequência maiores recusas alimentares. Porém, ainda são encontrados poucos artigos.
\end{abstract}

Palavras-chave: Transtorno do Espectro Autista; Seletividade alimentar; Criança; Comportamento alimentar.

\begin{abstract}
Introduction: Autism Spectrum Disorder (ASD) is considered a neurodevelopmental disorder that affects social communication and behaviors, which are restricted, persistent or stereotyped, including changes in sensory processing, in a hypo- or hyper-reactive way. These sensory dysfunctions can cause feeding problems, and the most cited food selectivity is common to children with ASD, where it is determined by food rejection, resistance to the new, and
\end{abstract}


limitation in the food repertoire. Objective: To identify sensory aspects and their interference in food selectivity of children with Autism Spectrum Disorder. Methods: It consists of an integrative review, using the following electronic databases: Lilacs (Literaturac Latino-Americana e do Caribe em Ciências da Saúde), Pubmed (U.S. National Library of Medicine) and Scielo (Scientific Electronic Library Online) by crossing the following descriptors, in Portuguese and English: Food selectivity/ Food Fussiness; Feeding Behavior/Autism Disorder; Autism Spectrum Disorder/Autism Spectrum Disorder; Autism/Autism; Autism/Autistic Disorder, using the Boolean operator AND. Results: A universe of 818 articles was obtained, after the eligible criteria 39 articles were selected. Finally, after reading the full articles, it resulted in 9 articles composing the study. Conclusion: The scientific literature demonstrates that children with Autism Spectrum Disorder present sensory alterations, such as: oral sensory sensitivity, tactile and olfactory. With the consequence of greater food refusals. However, few articles are found.

Keywords: Autistic Spectrum Disorder; Food fussiness; Child; Feeding behavior.

\section{Resumen}

Introducción: El trastorno del espectro autista (TEA) se considera un trastorno del neurodesarrollo que afecta a los medios de comunicación social y comportamientos, que son restringidos, persistentes o estereotipados, incluyendo los cambios en el procesamiento sensorial, de modo hipo o hiperreactivo. Estas disfunciones sensoriales pueden provocar problemas en la alimentación, siendo la selectividad alimentaria más citada común a niños con TEA, donde es determinada por el rechazo alimentario, resistencia al nuevo y limitación en el repertorio alimentario. Objetivo: Identificar los aspectos sensoriales y su interferencia en la selectividad alimentaria de los niños con Trastorno del Espectro Autista. Métodos: Consiste en una revisión integrativa, utilizando las siguientes bases de datos electrónica: Lilacs (Literaturac Latinoamericana y del Caribe en Ciencias de la Salud), PubMed (U.S. National Library of Medicine) y SciELO (Scientific Electronic Library Online) a través de cruce de los siguientes descriptores, en los idiomas portugués e inglés: Selectividad alimentaria/ Food Fussiness; Comportamiento alimentario/Feeding Behavior; Trastorno del Espectro Autista/Autism Spectrum Disorder; Autismo/Autism; Autismo infantil/Autistic Disorder, utilizando el operador booleano AND. Resultados: Se obtuvo un universo de 818 artículos, después de los criterios elegibles se seleccionaron 39 artículos. Finalmente, después de leer los artículos en su totalidad, resultó en 9 artículos componiendo el estudio. Conclusión: La literatura científica demuestra que los niños con Trastorno del Espectro Autista presentan alteraciones sensoriales, como: sensibilidad sensorial oral, táctil y olfativa. Teniendo como consecuencia mayores rechazos alimentarios. Sin embargo, aún se encuentran pocos artículos.

Palabras clave: Trastorno del Espectro Autista; Selectividad alimentaria; Niño; Comportamiento alimentario.

\section{Introdução}

O Transtorno do Espectro Autista (TEA) trata-se de um distúrbio do neurodesenvolvimento que apresenta déficits nas dimensões sociocomunicativas e comportamental de relação intrínseca, que se manifesta na infância (Santos \& Vieira, 2017). A concepção de espectro está associada a diversidade dos sintomas, que diferenciam em severidade, assim como a idade cronológica e o nível de desenvolvimento (Oliveira et al., 2018).

As características decorrentes do TEA, representam um continuum único de prejuízos correspondentes a intensidade que vão de leve a grave nas competências de comunicação social e de comportamentos restringidos e repetitivos (Nascimento et al., 2014).

Pesquisas apontaram que a prevalência do TEA no ano de 2015 pela Centers for Disease Control and Prevention (CDC) é de 14.7 por 1000 (1 a cada 45) em crianças com idade de 8 anos, acometendo 1 a cada 42 meninos e 1 a cada 189 meninas, na América do Norte (Caetano \& Gurgel, 2018). Acerca da etiopatogenia, ainda não foi completamente elucidada, no entanto, aponta-se forte envolvimento com relação multifatorial, entre diversas alterações genéticas, em conjuntos com os aspectos ambientais e biológicos (Monteiro, Santos, Gomes \& Rito, 2020).

No autismo podem ser observadas as seguintes manifestações: comportamentos restritos e persistentes, estereotipias motoras, ecolalias, frases idiossincráticas, inaptidão comunicativa, alterações do neurodesenvolvimento, comportamental ou mental; entre outras (Santos, 2019).

Dentre os sintomas apresentados no autismo, as dificuldades de comunicação têm maior visibilidade em comparação com as alterações sensoriais. Porém, as alterações sensoriais são bastante frequentes nessas crianças, porém, e geralmente passam despercebidas (Posar \& Visconti, 2018). 
Segundo Lázaro, Siquara e Pondé (2019), uma parcela significativa da comunidade autista apresentam problemas alimentares associados a disfunção sensorial, incompetências motoras orais relacionadas à mastigação e a deglutição e problemas no trato gastrointestinal (TGI). Os problemas referentes às alterações sensoriais, de modo hipo ou hiper-reativa, influenciam diretamente no paladar, olfato, audição, visão, sistema vestibular e propriocepção, de forma que é coerente considerar que esses problemas podem ser resultantes nas desordens comportamentais e alimentares de maneira direta ou indireta.

Os aspectos intrínsecos aos alimentos, são o sabor, textura, forma, temperatura dos alimentos, bem como a cor e o formato da embalagem, a apresentação do prato e dos utensílios empregues. A conduta de recusa desses fatores leva a formação dos hábitos alimentares, favorecendo um comer seletivo (Lázaro, Caron \& Pondé, 2018).

A seletividade alimentar (SA) é caracterizada pela tríade, que é composta pela rejeição alimentar, apetite diminuído e ausência de interesse pelo alimento. A combinação desses fatores pode promover uma restrição a variedades alimentares consumidos e extrema resistência em degustar novos alimentos. Essa limitação do repertório alimentar na hora da refeição, pode contribuir significantemente para escassez nutricional e provocar problemas no organismo. O comportamento de exclusão alimentar, pode muitas vezes ser transitório ou persistir ao longo do desenvolvimento da criança com autismo, já que elas são muito seletivas e resistentes ao novo (Rocha et al., 2019; Gomes et al., 2016).

Com base na literatura descrita, sobre o comportamento seletivo na alimentação envolvendo os aspectos sensoriais, surgiu a necessidade de compreender como se forma o comportamento alimentar das crianças que apresentam alterações sensoriais, contribuindo para um bom entendimento acerca dessas alterações e possibilitando auxílio aos pais e profissionais quanto a identificação destes fatores. Sendo assim, surge os seguintes questionamentos, quais são as alterações sensoriais de forma hipo e hiper-reativa que as crianças com espectro autista apresentam e se ocorre interferência alimentar a partir dessas alterações? Desta forma, o presente trabalho objetiva identificar os aspectos sensoriais e suas interferências na seletividade alimentar das crianças com Transtorno do Espectro Autista.

\section{Metodologia}

Trata-se de um estudo de revisão bibliográfica, do tipo integrativa, em virtude do objetivo que consiste em reunir, sintetizar e construir uma análise ampla dos resultados das pesquisas persistentes ao tema ou questão, de forma ordenada, favorecendo o conhecimento aprofundado sobre o tema pesquisado (Mendes, Silveira \& Galvão, 2008).

Representa um estudo de caráter exploratório, visto que proporciona maior conhecimento do problema, torna-o mais visível ou a constituir hipóteses. Tendo como objetivo principal da pesquisa, o aperfeiçoamento de idéias ou a descoberta de intuições (Gil, 2002). Se enquadrando como analítica, pois envolve uma análise mais complexa das informações coletadas de um determinado estudo, visando explicar a relação entre a causa e o efeito (Fontelles, Simões, Farias \& Fontelles, 2009), afim de verificar uma hipótese (Hochman, 2005).

As buscas foram iniciadas no período de março a abril de 2021, foram utilizados descritores na língua inglesa e portuguesa, através de consultas as bases de dados acerca de publicações referentes à temática abordada com leitura dos descritores, avaliação dos títulos, resumos e posteriormente a leitura completa dos artigos selecionados na íntegra, de acordo com os critérios de inclusão/exclusão pré-definidos. Para a pesquisa foram utilizadas as seguintes bases de dados: Literaturac LatinoAmericana e do Caribe em Ciências da Saúde (LILACS), Us national library of Medicine National Institute of Health (PubMED) e Scientific Electronic Library Online (Scielo).

A busca dos artigos ocorreu através de uma análise e combinações dos descritores pela a plataforma de Descritores em Ciências da Saúde (DeCS), havendo interligação com o operador booleano AND, sendo assim definidos conforme apresentados na Tabela 1.

Na Tabela 1, apresenta os descritores e os cruzamentos para identificação dos artigos, com base no DeCS. 
Research, Society and Development, v. 10, n. 10, e557101018944, 2021

(CC BY 4.0) | ISSN 2525-3409 | DOI: http://dx.doi.org/10.33448/rsd-v10i10.18944

Tabela 1 - Apresentação dos descritores com base no DeCS, e os seus cruzamentos.

\begin{tabular}{|c|c|c|}
\hline \multicolumn{3}{|c|}{ Cruzamento dos descritores } \\
\hline Descritores & $\begin{array}{c}\text { Cruzamento dos descritores em } \\
\text { Português }\end{array}$ & $\begin{array}{c}\text { Cruzamento dos descritores } \\
\text { em Inglês }\end{array}$ \\
\hline $\begin{array}{l}\text { 1. Seletividade } \\
\text { Alimentar }\end{array}$ & Autismo e Seletividade Alimentar & Autism and Food Fussiness \\
\hline $\begin{array}{l}\text { 2. Comportamento } \\
\text { Alimentar }\end{array}$ & Autismo e Comportamento Alimentar & Autism and Feeding Behavior \\
\hline $\begin{array}{l}\text { 3. Transtorno do } \\
\text { Espectro Autista }\end{array}$ & $\begin{array}{l}\text { Seletividade Alimentar e Transtorno do } \\
\text { Espectro Autista }\end{array}$ & $\begin{array}{l}\text { Food Fussiness and Autism } \\
\text { Spectrum Disorder }\end{array}$ \\
\hline 4. Autismo & $\begin{array}{l}\text { Comportamento Alimentar e Transtorno } \\
\text { do Espectro Autista; }\end{array}$ & $\begin{array}{l}\text { Feeding Behavior and Autism } \\
\text { Spectrum Disorder }\end{array}$ \\
\hline 5. Autismo Infantil & $\begin{array}{c}\text { Autismo infantil e Comportamento } \\
\text { Alimentar; }\end{array}$ & $\begin{array}{l}\text { Autistic Disorder and } \\
\text { Feeding Behavior }\end{array}$ \\
\hline & $\begin{array}{c}\text { Autismo Infantil e Seletividade } \\
\text { Alimentar }\end{array}$ & $\begin{array}{l}\text { Autistic Disorder and Food } \\
\text { Fussiness. }\end{array}$ \\
\hline
\end{tabular}

Fonte: Dados da pesquisa (2021).

Os critérios de inclusão foram: artigos em português e inglês, originais na íntegra, que abordassem a temática; o tempo de publicação entre 2011 a 2021, tendo em vista a atuação fonoaudiológica em meio a seletividade alimentar em crianças com TEA. Foram excluídos os artigos com crianças autistas com comorbidades associadas que levem à alteração sensorial e crianças com a faixa etária maior que 12 anos.

As buscas foram realizadas nas seguintes bases de dados: LILACS, PubMed e Scielo.

A análise de dados ocorreu pela categorização sistemática, através de uma planilha no Microsoft Excel, sendo organizadas em eixos temáticos para melhor análise e interpretação dos dados. Os eixos temáticos foram: a) autor/ano; b) título; c) amostra; d) idade; e) base de dados; f) objetivo; g) protocolos utilizados para classificação das crianças autistas com alterações sensoriais que interferem no comportamento alimentar/seletividade alimentar; h) presença de alteração sensorial; i) tipo de alteração sensorial; j) contextualização. Os dados obtidos foram distribuídos em duas partes: a) Aspectos metodológicos, sendo aplicados em uma tabela; b) Análise dos estudos, em mapa conceitual. Quanto à análise dos dados, utilizou-se a estatística descritiva com o objetivo de organizar, descrever, resumir e apresentar os resultados.

Nesta revisão integrativa, o número total de artigos selecionado foi 818, sendo 785 da plataforma PubMed, 25 do LILACS e 8 da Scielo. O fluxograma da identificação dos artigos, está exibido na Figura 1. 
Figura 1 - Fluxograma do resultado do processo de seleção dos artigos, segundo base de dados.
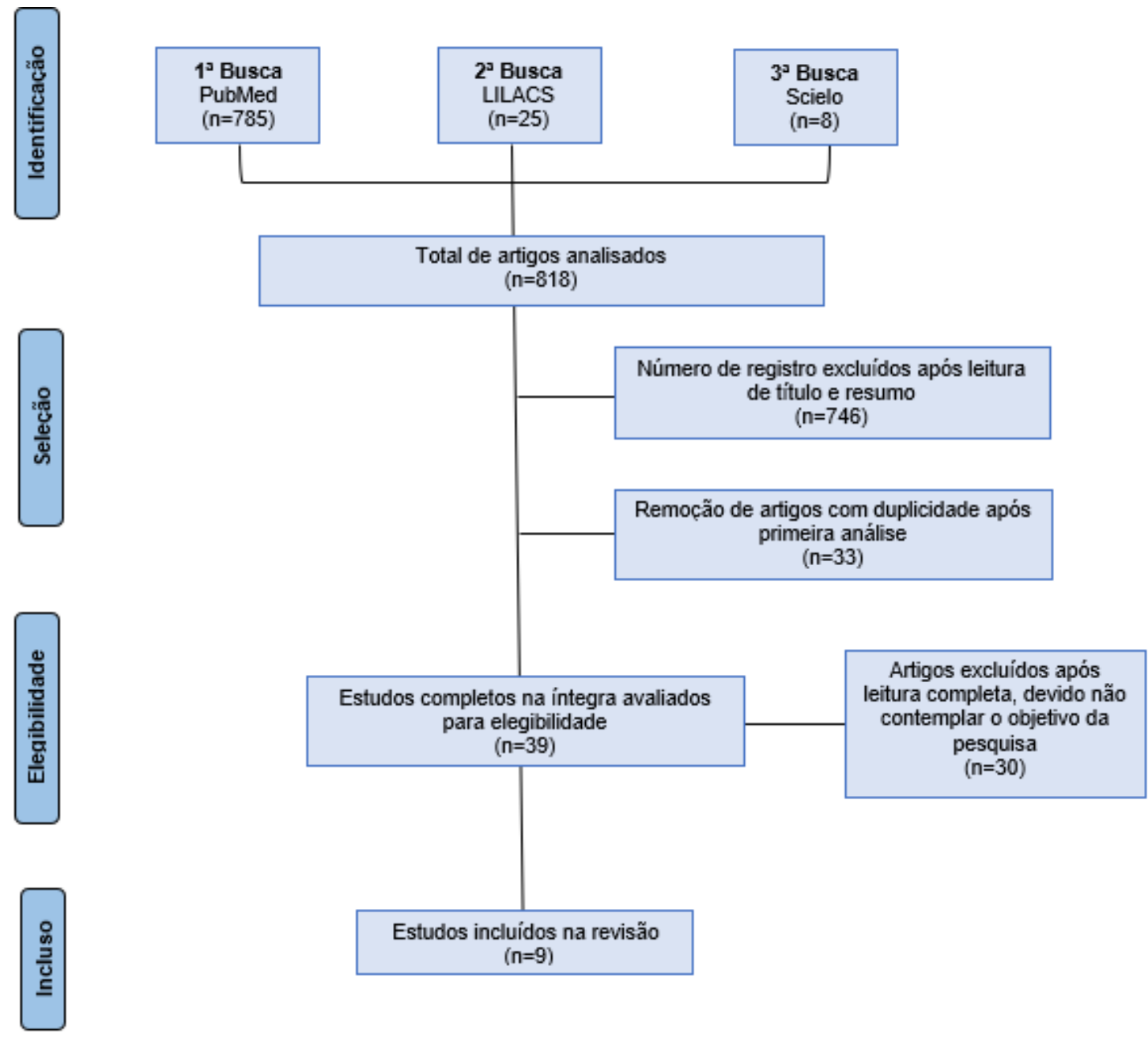

Fonte: Dados da pesquisa (2021).

A seleção foi realizada com base nos critérios elegíveis. A busca inicial resultou em 818 artigos. Desses, foram excluídos 746 após leitura de títulos e resumos, 33 artigos por repetição após a primeira análise. Resultou em 39 estudos elegíveis e desses foram excluídos 30, após leitura do texto completa e por não atender o objetivo proposto na pesquisa. A seleção final totalizou em 9 artigos para compor a presente revisão.

\section{Resultados}

Com base nos 9 estudos selecionados e analisados, foram extraídos deles os seguintes dados: a) Autor/ano; b) Título; c) Amostra; d) Descritores; e) Base de dados, como mostra abaixo a Tabela 2. 
Tabela 2 - Relação dos estudos selecionados.

\begin{tabular}{|c|c|c|c|c|c|}
\hline $\mathbf{N}^{o}$ & Autor/Ano & Título & Amostra (n) & Descritores & $\begin{array}{c}\text { Base de } \\
\text { Dados }\end{array}$ \\
\hline 1 & $\begin{array}{c}\text { Johnson et al., } \\
2014\end{array}$ & $\begin{array}{c}\text { Relationships Between Feeding } \\
\text { Problems, Behavioral } \\
\text { Characteristics and Nutritional } \\
\text { Quality in Children with ASD }\end{array}$ & $\begin{array}{l}256 \text { crianças } \\
\text { com TEA }\end{array}$ & $\begin{array}{l}\text { Feeding problems; } \\
\text { Mealtime; Nutrition }\end{array}$ & PubMed \\
\hline 2 & $\begin{array}{l}\text { Hubbard, } \\
\text { Anderson, } \\
\text { Curtin, Must e } \\
\text { Bandini (2014) }\end{array}$ & $\begin{array}{l}\text { A comparison of food refusal } \\
\text { related to characteristics of } \\
\text { food in children with autism } \\
\text { spectrum disorder and typically } \\
\text { developing children }\end{array}$ & $\begin{array}{l}53 \text { crianças } \\
\text { com TEA e } 58 \\
\text { com DT }\end{array}$ & $\begin{array}{l}\text { Autism spectrum } \\
\text { disorder; Children; } \\
\text { Feeding; Fruit and } \\
\text { vegetable intake; } \\
\text { Nutrition. }\end{array}$ & PubMed \\
\hline 3 & $\begin{array}{c}\text { Tanner et al., } \\
2015 .\end{array}$ & $\begin{array}{l}\text { Behavioral and Physiological } \\
\text { Factors Associated With } \\
\text { Selective Eating in Children } \\
\text { With Autism Spectrum } \\
\text { Disorder }\end{array}$ & $\begin{array}{l}35 \text { pais de } \\
\text { crianças com } \\
\text { TEA }\end{array}$ & $\begin{array}{l}\text { Child behavior, child } \\
\text { development } \\
\text { disorders, pervasive, } \\
\text { eating, feeding } \\
\text { behavior, food } \\
\text { habits, sensation }\end{array}$ & PubMed \\
\hline 4 & $\begin{array}{l}\text { Marshall et al., } \\
2015 .\end{array}$ & $\begin{array}{l}\text { Multidisciplinary Intervention } \\
\text { for Childhood Feeding } \\
\text { Difficulties }\end{array}$ & $\begin{array}{l}33 \text { crianças } \\
\text { com TEA e } 35 \\
\text { com NMC }\end{array}$ & $\begin{array}{c}\text { Autism spectrum } \\
\text { disorders; feeding } \\
\text { difficulties; } \\
\text { intervention; } \\
\text { nutrition; weight }\end{array}$ & PubMed \\
\hline 5 & $\begin{array}{l}\text { Marshall et al., } \\
2016 .\end{array}$ & $\begin{array}{l}\text { Clinical Characteristics of } 2 \\
\text { Groups of Children With } \\
\text { Feeding Difficulties }\end{array}$ & $\begin{array}{l}33 \text { crianças } \\
\text { com TEA e } 35 \\
\text { com uma } \\
\text { história não } \\
\text { clinicamente } \\
\text { complexa } \\
\text { (NMC) }\end{array}$ & $\begin{array}{l}\text { Autism spectrum } \\
\text { disorders; feeding } \\
\text { difficulties; } \\
\text { nonmedically } \\
\text { complex children; } \\
\text { nonorganic failure to } \\
\text { thrive; nutrition }\end{array}$ & PubMed \\
\hline 6 & $\begin{array}{c}\text { Chistol et al., } \\
2018\end{array}$ & $\begin{array}{l}\text { Sensory sensitivity and food } \\
\text { selectivity in children with } \\
\text { autism spectrum disorder }\end{array}$ & $\begin{array}{l}53 \text { crianças } \\
\text { com TEA e } 56 \\
\text { com DT }\end{array}$ & $\begin{array}{l}\text { Autism spectrum } \\
\text { disorder; Food } \\
\text { selectivity; Sensory } \\
\text { sensitivity. }\end{array}$ & PubMed \\
\hline 7 & $\begin{array}{l}\text { Seiverling et al. } \\
\text { (2018). }\end{array}$ & $\begin{array}{c}\text { A Comparison of a Behavioral } \\
\text { Feeding Intervention With and } \\
\text { Without Pre-meal Sensory } \\
\text { Integration Therapy }\end{array}$ & $\begin{array}{c}2 \text { crianças com } \\
\text { TEA }\end{array}$ & $\begin{array}{l}\text { Behavioral feeding } \\
\text { intervention; Food } \\
\text { selectivity; Sensory } \\
\text { integration therapy. }\end{array}$ & $\begin{array}{l}\text { SEIVERLING } \\
\text { et al., } 2018 .\end{array}$ \\
\hline 8 & $\begin{array}{l}\text { Ismail, Ramli, } \\
\text { Hamzaid e } \\
\text { Hassan, } 2020 .\end{array}$ & $\begin{array}{l}\text { Exploring Eating and } \\
\text { Nutritional Challenges for } \\
\text { Children with Autism } \\
\text { Spectrum Disorder: Parents' } \\
\text { and Special Educators' } \\
\text { Perceptions }\end{array}$ & $\begin{array}{c}14 \text { pais e } 6 \\
\text { educadores } \\
\text { especiais }\end{array}$ & $\begin{array}{l}\text { ASD; education; } \\
\text { food behaviour; } \\
\text { module; nutrition }\end{array}$ & PubMed \\
\hline 9 & $\begin{array}{c}\text { Oliveira e } \\
\text { Frutuoso, } 2021 .\end{array}$ & $\begin{array}{l}\text { Muito além dos nutrientes: } \\
\text { experiências e conexões com } \\
\text { crianças autistas a partir do } \\
\text { cozinhar e comer juntos }\end{array}$ & $\begin{array}{l}17 \text { crianças e } \\
\text { adolescentes }\end{array}$ & $\begin{array}{c}\text { Nutrição da Criança; } \\
\text { Culinária; } \\
\text { Transtorno do } \\
\text { Espectro Autista; } \\
\text { Relações } \\
\text { Interpessoais }\end{array}$ & Scielo \\
\hline
\end{tabular}

Fonte: Dados da pesquisa (2021).

Na Tabela 2, mostra que na pesquisa, os anos das publicações dos estudos encontrados variou de 2014 a 2021 , sendo dois artigos de 2014 e 2015, um de 2016, dois de 2018 e um de 2020 e 2021. Desses nove artigos, oito estavam em inglês e se encontravam na plataforma PubMed e um em português e localizado na Scielo. Todos os estudos abordaram crianças com TEA.

Na Figura 2 abaixo, é apresentado o mapa conceitual, que dispõe da análise dos estudos encontrados com base na seletividade alimentar em crianças autistas. Sendo ela determinada pela repulsão de novos alimentos, que contribuem para um 
repertório alimentar limitado com um número baixo de alimentos (Pereira, 2019), obtendo a sensorialidade como uma das causas para o surgimento.

São apresentadas no mapa, as características dos artigos selecionados, contendo: idade, objetivo, protocolo utilizado, tipo de alteração sensorial e contextualização.

Figura 2 - Mapa conceitual dos achados científicos de Seletividade Alimentar em crianças com TEA.

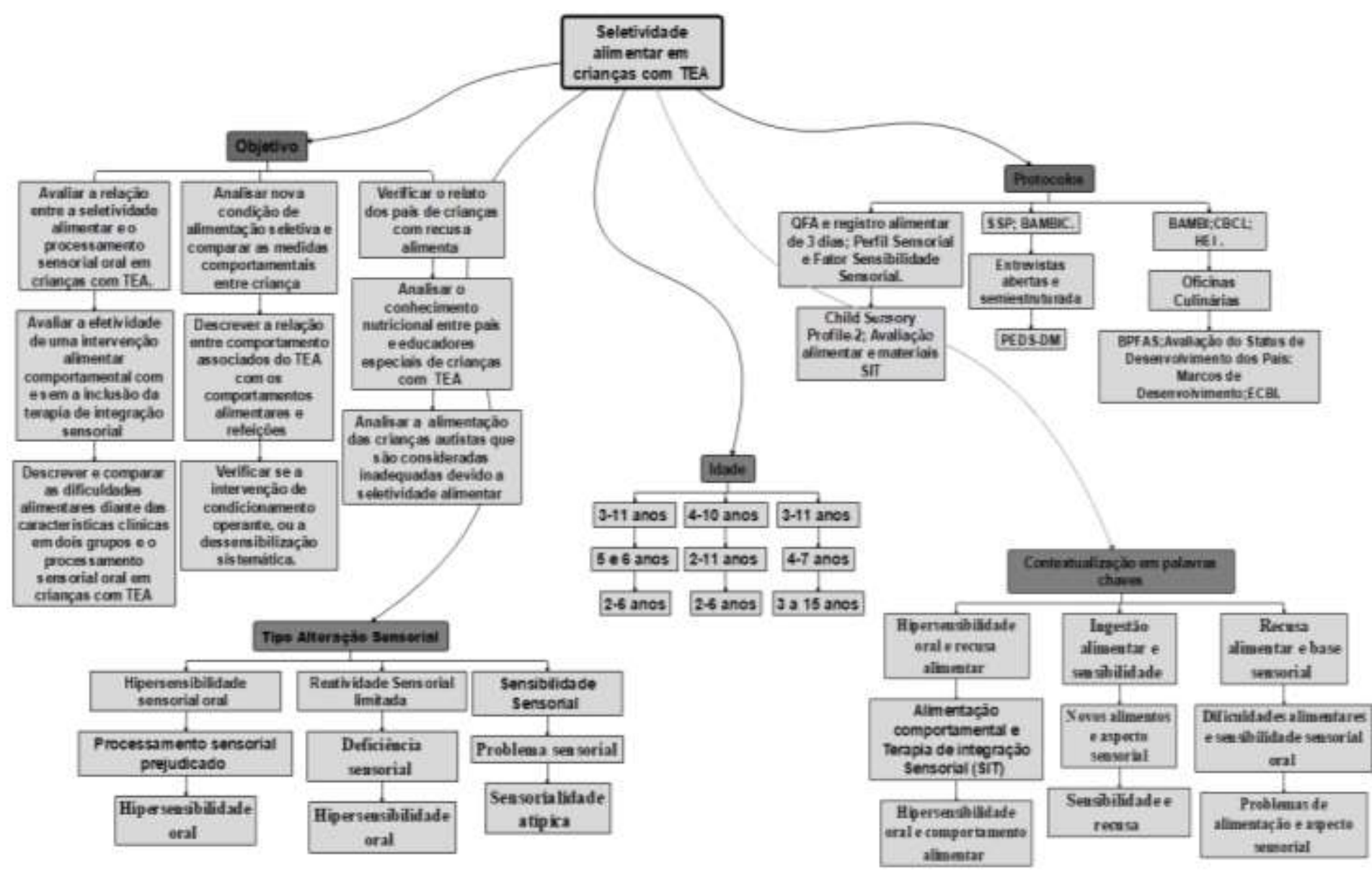

Fonte: Dados da pesquisa (2021).

A idade das crianças avaliadas nos estudos foram entre 2 a 11 anos, porém, foi encontrado um artigo que abordava crianças até 15 anos. Na presente revisão foi coletado somente os dados avaliativos das crianças até 12 anos, pois conforme diz o art. $2^{\circ}$ do Estatuto da Criança e do Adolescente, (Lei n.8.069, de 13 de julho de 1990), considera-se criança toda as pessoas com até 12 anos incompletos (Brasil, 1990).

Foi observado que os protocolos utilizados para identificação das crianças autistas com alterações sensoriais que interferiam no comportamento alimentar se repetiam. Os protocolos utilizados foram: Questionário de Frequência Alimentar (QFA), Questionário Perfil Sensorial e Fator Sensibilidade Sensorial, Perfil Sensorial Curto (SSP), Avaliação Breve do Comportamento das Refeições em Crianças (BAMBIC), Child Sensory Profile-2 (CSP-2), Avaliação alimentar através de alimentos alvos e materiais da terapia de integração sensorial (SIT), Brief Autism Mealtime Behavior Inventory (BAMBI), Child Behavior Checklist (CBCL), O Índice de Alimentação Saudável (HEI), Entrevista semiestruturada, Avaliação de Alimentação de Pediatria Comportamental (BPFAS); Avaliação do Status de Desenvolvimento dos Pais: Marcos de Desenvolvimento, Eyberg Child Behavior Inventory (ECBI) e Oficinas Culinárias (Johnson et al., 2014; Hubbard, Anderson, Curtin, Must \& Bandini, 2014; 
Tanner et al., 2015; Marshall et al., 2015; Marshall et al., 2016; Chistol et al., 2018; Seiverling et al., 2018; Ismail, Ramli, Hamzaid \& Hassan, 2020; Oliveira e Frutuoso, 2021).

Os estudos tiveram como objetivo analisar, descrever, verificar, avaliar a seletividade, o comportamento, a recusa alimentar das crianças com TEA e a relação sensorial. Como apresentado no mapa, é possível identificar que todos os estudos apontaram uma alteração no processamento sensorial, com base no tipo de alteração e na contextualização.

A contextualização do presente estudo foi abordada em palavras-chaves com a finalidade de síntese nos achados, encontrando assim, as principais características: sensibilidade sensorial oral, comportamento alimentar, recusa alimentar e dificuldades alimentares.

\section{Discussão}

De acordo com os estudos analisados, as crianças com TEA são seletivas e apresentam menor aceitação na ingestão de novos alimentos em comparação com as crianças com desenvolvimento típico, tendo como características a recusa de alimentos, problemas sensoriais e comportamentos difíceis na refeição, sendo justificados por outra revisão de literatura (Almeida, 2020) e discorridos nesse estudo.

Sendo assim, em uma pesquisa comparativa, obteve-se que crianças com TEA recusaram mais alimentos, apresentaram hipersensibilidade sensorial atípica e são mais seletivas em comparação com as crianças típicas. A recusa alimentar foi mais evidenciada pela textura, consistência/sabor e cheiro. Esses achados são condizentes com a pesquisa de Rocha et al. (2019), na qual $75 \%$ das crianças autistas tem preferência alimentar através da textura, cor e cheiro, e 68,9\% costumam recusar alimentos, com maior rejeição às categorias de frutas, vegetais, leites e derivados.

Além das recusas alimentares relacionadas à textura, cor e cheiro, como também rejeição das categorias alimentares mencionadas, algumas atitudes como cheirar e brincar com o alimento, recusar-se a tocar, lamber e não comer, apontaram sensorialidade presente nas crianças com TEA. Esses resultados assemelham-se com os estudos que demonstram que a seletividade e às recusas alimentares, possivelmente estão associados à um problema sensorial (Posar e Visconti, 2018; Rodrigues et al., 2020), sendo a textura o fator mais relacionado a seletividade (Zobel-Lachiusa, Andrianopoulos, Mailloux \& Cermak 2015; Almeida et al., 2018)

Com base na relação entre a SA e recusas alimentares com as alterações sensoriais, compreende-se que o processamento sensorial possui a capacidade de organizar, perceber e interpretar as informações recebidas através dos sistemas sensoriais, como paladar, olfato, tato, vestibular e auditivo, produzindo uma resposta adaptativa (Kilroy \& Aziz - Zadeh \& Cermak, 2019).

E quando existem problemas no funcionamento sensorial, podem haver alterações no processo alimentar, devido à alimentação ter uma base bastante sensorial, sendo realizado na boca, uma das áreas mais sensitivas do corpo humano, segundo Figueira (2017), que corrobora com três estudos apresentados nessa revisão sobre as dificuldades alimentares e aspectos sensoriais (Johnson et al., 2014; Marshall, Hill, Ware. Ziviani \& Dobrill, 2015; Marshall et al., 2016).

Seiverling et al. (2018) afirmaram que as abordagens de alimentação comportamental são mais satisfatórias do que a fundamentada nos sentidos, tendo os seus achados consistentes ao que referem Gama et al., (2020), que declara que poucos foram os artigos encontrados sobre integração sensorial e tratamento em SA, determinando a insuficiência de dados sobre a prática, como realizar os métodos e utilizar técnicas que favoreçam a redução do comer seletivo em crianças com TEA. Porém, mesmo com poucos estudos de revisão bibliográfica, ainda demonstra-se que a intervenção precoce no processamento sensorial, ajuda na diminuição desse problema e auxilia na seletividade alimentar em crianças autistas. 
Research, Society and Development, v. 10, n. 10, e557101018944, 2021

(CC BY 4.0) | ISSN 2525-3409 | DOI: http://dx.doi.org/10.33448/rsd-v10i10.18944

\section{Conclusão}

A pesquisa aponta que existe relação entre seletividade alimentar e alterações sensoriais nas crianças com TEA. As alterações sensoriais identificadas foram: sensibilidade sensorial oral, sensibilidade sensorial tátil e sensibilidade sensorial olfativa. A sensibilidade sensorial composta por recusa a ingerir alimentos devido a sua textura e sabor. A sensibilidade sensorial tátil, devido a recusa-se a tocar e por a sensibilidade sensorial olfativa, cheirar os alimentos e não consumir. Essas crianças apresentaram mais problemas na alimentação e maiores comportamentos de recusa. Porém, ainda são encontrados poucos estudos, nesta área.

Faz-se necessário um aprofundamento na abordagem para avaliar a seletividade alimentar e a sensorialidade, principalmente em crianças autistas, pois é mais comumente encontrada a SA nelas do que em crianças com desenvolvimento típico. Sendo primordial a atuação da equipe multiprofissional, com a finalidade de ampliar o conhecimento sobre o assunto, trazendo maiores resultados de pesquisa no campo cientifico.

\section{Referências}

Almeida, B. F. P (2020). Autismo, seletividade alimentar e transtorno do processamento sensorial: revisão de literatura. Monografia de Especialização

Almeida, A. K. A., Fonseca, P. C. A, Oliveira, L. A., Santos, W. R. C. C., Zagmignan, A., de Oliveira, B. R., \& de Carvalho, C. A. (2018). Consumo de ultraprocessados e estado nutricional de crianças com transtorno do espectro do autismo. Revista Brasileira em Promoção da Saúde, 31(3).

Brasil. (1990). Lei no 8.069, de 13 de julho de 1990. Dispõe sobre o Estatuto da Criança e do Adolescente e dá outras providências. Diário Oficial da República Federativa do Brasil

Caetano, M. V., \& Gurgel, D. C. (2018). Nutritional profile of children bearing autism spectrum disorder. Revista Brasileira em Promocao da Saude, 31(1).

Chistol, L. T., Bandini, L. G., Must, A., Phillips, S., Cermak, S. A., \& Curtin, C. (2018). Sensory sensitivity and food selectivity in children with autism spectrum disorder. Journal of autism and developmental disorders, 48(2), 583-591.

Figueira, O. (2017). Alimentação e funcionamento sensorial em crianças de cinco anos (Doctoral dissertation).

Fontelles, M. J., Simões, M. G., Farias, S. H., \& Fontelles, R. G. S. (2009). Metodologia da pesquisa científica: diretrizes para a elaboração de um protocolo de pesquisa. Revista paraense de medicina, 23(3), 1-8.

Gama, B. T. B., Lobo, H. H. M., da Silva, A. K. T., \& Montenegro, K. S. (2020). Seletividade alimentar em crianças com Transtorno do Espectro Autista (TEA): uma revisão narrativa da literatura. Revista Artigos. Com, 17, e3916-e3916.

Gil, A. C. (2002). Como elaborar projetos de pesquisa (Vol. 4, p. 175). São Paulo: Atlas.

Gomes, V. T. S., Gomes, R. N. S., Gomes, M. S., Viana, L. V. M., Conceição, F. R., Amorim, L. M. M., \& Soares, E. L. (2016). Nutrição e autismo: reflexões sobre a alimentação do autista. Revista Univap, São José dos Campos, 22(40).

Hubbard, K. L., Anderson, S. E., Curtin, C., Must, A., \& Bandini, L. G. (2014). A comparison of food refusal related to characteristics of food in children with autism spectrum disorder and typically developing children. Journal of the Academy of Nutrition and Dietetics, 114(12), $1981-1987$.

Hochman, B., Nahas, F. X., Oliveira Filho, R. S. D., \& Ferreira, L. M. (2005). Desenhos de pesquisa. Acta Cirúrgica Brasileira, $20,2-9$.

Ismail, N. A. S., Ramli, N. S., Hamzaid, N. H., \& Hassan, N. I. (2020). Exploring Eating and Nutritional Challenges for Children with Autism Spectrum Disorder: Parents' and Special Educators' Perceptions. Nutrients, 12(9), p. 2530.

Johnson, C. R., Turner, K., Stewart, P. A., Schmidt, B., Shui, A., Macklin, E., \& Hyman, S. L. (2014). Relationships between feeding problems, behavioral characteristics and nutritional quality in children with ASD. Journal of autism and developmental disorders, 44(9), $2175-2184$.

Kilroy, E., Aziz-Zadeh, L., \& Cermak, S. (2019). Ayres theories of autism and sensory integration revisited: What contemporary neuroscience has to say. Brain sciences, 9(3), p. 68.

Lázaro, C. P., Caron, J., \& Pondé, M. P. (2018). Scales assessing eating behavior in autism spectrum disorder. Psicologia: teoria e prática, $20(3)$, $42-59$.

Lázaro, C. P., Siquara, G. M., \& Pondé, M. P. (2020). Escala de Avaliação do Comportamento Alimentar no Transtorno do Espectro Autista: estudo de validação. Jornal Brasileiro de Psiquiatria, 68, 191-199.

Marshall, J., Hill, R. J., Ware, R. S., Ziviani, J., \& Dodrill, P. (2016). Clinical characteristics of 2 groups of children with feeding difficulties. Journal of pediatric gastroenterology and nutrition, 62(1), 161-168.

Marshall, J., Hill, R. J., Ware, R. S., Ziviani, J., \& Dodrill, P. (2015). Multidisciplinary intervention for childhood feeding difficulties. Journal of pediatric gastroenterology and nutrition, 60(5), 680-687. 
Mendes, K. D. S., Silveira, R. C. D. C. P., \& Galvão, C. M. (2008). Revisão integrativa: método de pesquisa para a incorporação de evidências na saúde e na enfermagem. Texto \& contexto-enfermagem, 17, 758-764.

Monteiro, M. A., Santos, A. A. A. D., Gomes, L. M. M., \& Rito, R. V. V. F. (2020). Transtorno do espectro autista: uma revisão sistemática sobre medidas nutricionais. Revista Paulista de Pediatria, 38.

Nascimento, M. I. C., Machado, P. H., Garcez, R. M., Pizzato, R., Rosa, S. M. M. D., American Psychiatric Association, \& American Psychiatric Association. (2014). Manual diagnóstico e estatístico de transtornos mentais: DSM-5. Associação Brasileira de Psiquiatria. Porto Alegre: Artmed, p. 42.

Oliveira, L. M. M., Maia, F. A., Almeida, M. T. C., Alves, M. R., Lopes, L. V. S. B., Oliveira, V. S. D., \& Silveira, M. F. (2018). Fatores pós-natais relacionados ao transtorno do espectro do autismo: revisão integrativa da literatura. Unimontes Científica, 20(1), 2-22.

Oliveira, B. M. F. D., \& Frutuoso, M. F. P. (2021). Muito além dos nutrientes: experiências e conexões com crianças autistas a partir do cozinhar e comer juntos. Cadernos de Saúde Pública, 37, e00132020.

Pereira, A. D. S. (2019). Comportamento alimentar de crianças com transtorno do espectro autista (TEA) (Bachelor's thesis).

Posar, A., \& Visconti, P. (2018). Sensory abnormalities in children with autism spectrum disorder. Jornal de pediatria, $94,342-350$.

Rodrigues, C. P. S., de Araújo Silva, J. P., Álvares, I. Q., Silva, A. L. F., Leite, A. F. B., \& Carvalho, M. F. (2020). O consumo alimentar de crianças com Transtorno do Espectro Autista está correlacionado com alterações sensório-oral e o comportamento alimentar. Brazilian Journal of Development, 6(9), 6715567170 .

Rocha, G. S. S., de Medeiros Júnior, F. C., Lima, N. D. P., da Silva Machado, A., Pereira, I. C., da Silva Lima, M., \& da Silva, H. A. C. (2019). Análise da seletividade alimentar de pessoas com Transtorno do Espectro Autista. Revista Eletrônica Acervo Saúde, (24), e538-e538.

Santos, R. K., \& da Silva, A. M. E. C. (2017). Transtorno Do Espectro Do Autismo (Tea): Do Reconhecimento À Inclusão No Âmbito Educacional. Revista Includere, 3(1).

Santos, B. (2020). Critério Diagnóstico Fonoaudiológico No Transtorno Do Espectro Autista: Revisão De Literatura. Revista GepesVida, 5(13).

Seiverling, L., Anderson, K., Rogan, C., Alaimo, C., Argott, P., \& Panora, J. (2018). A comparison of a behavioral feeding intervention with and without premeal sensory integration therapy. Journal of autism and developmental disorders, 48(10), 3344-3353.

Tanner, K., Case-Smith, J., Nahikian-Nelms, M., Ratliff-Schaub, K., Spees, C., \& Darragh, A. R. (2015). Behavioral and physiological factors associated with selective eating in children with autism spectrum disorder. American Journal of Occupational Therapy, 69(6), 6906180030p1-6906180030p8.

Zobel-Lachiusa, J., Andrianopoulos, M. V., Mailloux, Z., \& Cermak, S. A. (2015). Sensory differences and mealtime behavior in children with autism. American Journal of Occupational Therapy, 69(5), 6905185050p1-6905185050p8. 